\title{
Preface to the special issue of INCAM 2019 conference on solid mechanics
}

\author{
Ratna Kumar Annabattula ${ }^{1}$ S. Pradyumna
}

Published online: 31 July 2020

(C) Indian Institute of Technology Madras 2020

Indian Conference on Applied Mechanics (INCAM) is the flagship event of the Indian Society of Applied Mechanics (ISAM). INCAM is a biennial event that brings together the students and researchers from academia and industry working in various aspects of applied mechanics.

The fourth Indian Conference on Applied Mechanics (INCAM-2019) was jointly organized by the Department of Civil Engineering and Mechanical Engineering at the Indian Institute of Science Bengaluru during July 3-5, 2019. Of the 57 presentations in the area of solid mechanics, 21 authors have been invited to submit full papers for consideration. Of the 21 invitations, 14 authors have submitted the full papers, and finally, 9 of them have been accepted for publication. The articles have been thoroughly peer-reviewed by experts in the respective area. From the 9 accepted papers, 7 articles are published in the current issue and the remaining two articles will appear in the next issue due to unforeseen delays. The papers published in this special issue cover topics on mechanics of composite and elastomer materials, contact mechanics, damage mechanics, and fracture mechanics. A brief overview of the articles is presented in the same order as they appear in the special issue.

1. Continuum damage-healing based constitutive modelling for self-healing materials-Application to one dimensional cyclic loading cases In this work, the

Ratna Kumar Annabattula ratna@iitm.ac.in

1 Department of Mechanical Engineering, Indian Institute of Technology Madras, Chennai, India

2 Department of Applied Mechanics, Indian Institute of Technology, New Delhi, Delhi, India authors report a continuum damage-healing mechanics (CDHM) model to study the self-healing behaviour of a class of materials that undergo self-healing at lower strains values.

2. Influence of crack driving force on correlating stress ratio effects in fatigue crack growth rate of a Nickelbased superalloy IN720 In this experimental work, the authors report the effect of stress ratio on the fatigue crack growth rate in a Ni-based superalloy IN720.

3. Bending analysis of laminated beams using Isogeometric variational asymptotic method In this article, the authors use Isogeometric variational asymptotic method to study the bending deformation of laminated composite beams.

4. Numerical Analysis of ball-pitch impact in Cricket In this paper, a finite element model describing the impact of a cricket ball on the pitch is presented. The authors model the pitch as a two-layer composite and discuss the influence of various parameters on the pitch behaviour and the ball dynamics.

5. Lumped Parameter Models for Adhesive Contact Mechanics The authors report two lumped parameter models to compute the adhesive forces acting on the contact zone to reduce the computational cost of a conventional finite element simulation.

6. A 3D Solution for Angle-ply Cylindrical Shell Panel Supported Arbitrarily on its Boundaries using Extended Kantorovich Method In this paper, the authors present a generalized three-dimensional solution for an angle-ply cylindrical shell panel using the multi-term Extended Kantorovich Method (EKM). The authors also demonstrate the efficacy of the method over conventional 3D finite element solution. 
7. A wedge penetration model to estimate leak through elastomer-metal interface The paper presents a predictive model to understand the failure of seals used in various engineering assemblies for leak proofing. The authors present a generic framework which is capable of predicting gradual as well as complete failure of seals used in elastomer-metal interfaces.

We appreciate the authors for their excellent support and coming forward to contribute to this special issue. We thank all the reviewers for their time and effort, thus helping us make the right decision about the papers' quality. The Springer publishing team has been very helpful in setting up the portal and answering all the queries promptly. We thank the Editor-in-Chief Prof. Harishankar Ramachandran, for his support in bringing this special issue. 